\title{
Learning Basic Dance Choreographies with different Augmented Feedback Modalities
}

Dieter Drobny

RWTH Aachen University

52056 Aachen, Germany

dieter.drobny@rwth-aachen.de

Jan Borchers

RWTH Aachen University

52056 Aachen, Germany

borchers@cs.rwth-aachen.de

\begin{abstract}
We plan to evaluate different kinds of augmented feedback (tactile, video, sound) for learning basic dance choreographies. Therefore we develop a dance training system based on motion capturing technology. In this work we describe and put up for discussion its capabilities and our methodological approach.
\end{abstract}

\section{Authors Keywords}

Dancing, wireless sensor system, motor skill learning, tactile feedback.

\section{ACM Classification Keywords}

C. 3 [Special-purpose and application-based systems]: Real-Time and embedded system; H.5.2 [Information Interfaces and Representations - User Interfaces]: a - Auditory feedback, Haptic I/O,

p - Training, help, and documentation.

\section{General Terms}

Experimentation

\section{Introduction and Motivation}

CHI 2010, April 10-15, 2010, Atlanta, Georgia, USA. ACM 978-1-60558-930-5/10/04.
Learning physical activities, such as dancing or many sports, is normally done in a teacher-student(s) relationship with very little use of other methods than 
verbal instructions from the teacher, her demonstration of movements, or her physical guidance of a student's movement or posture.

During typical beginner courses, students learn a simple choreography to train basic steps and to enhance their capabilities to dance in sync to the music. The Aerobic, Hip-Hop, Jazz, or Rock'n'Roll courses of our university, e.g., use this approach. In these beginner courses much of the time is devoted to teach the correct sequence of movements without focusing on details. In following courses the focus shifts more and more towards detailed movement corrections, such as how a specific step or an arm movement should be performed. We focus on support for dancers of beginner courses.

\section{Definition}

In the following, we differentiate between support and feedback. We define support as any kind of help that does not depend on a student's performance. For example, repeated demonstrations of a video with a dancer performing a choreography is a supporting function. Feedback, however, depends on a student's performance. If just one part of a choreography with which a student has problems is explained again if problems have been detected, this is not a simple supporting function anymore as the decision which part is repeated depends on the student's performance. In some cases the difference between a supporting function and feedback is not so obvious, in cases of concurrent feedback (which is activated or deactivated automatically) the difference to a simple supporting functions (which is turned on all the time) becomes more obvious.

\section{Related Work}

Nakamura et. al.'s [1] Multimodal Presentation method for a Dance Training System supports learners of a traditional Japanese folk dance by giving vibrotactile cues 0.5 seconds prior expected arm movements. In a small experiment they evaluated performance under two conditions: Video plus vibrotactile cues and video without vibrotactile cues. They considered a movement as correct if it was executed within a one second timeframe. We believe that this timeframe was chosen too big to represent correct movements in typical dances. In most dances, a step is performed on at least each beat of the music. Even in slow dances like Slow Waltz, which is danced to music with about 86 beats per minute, this means one step every 0.7 seconds.

Spelmezan et. al. [2] use vibrational devices to support beginners of snowboarding. In contrast to Nakamura's work, their system includes sensors and can dynamically adapt the vibrotactile feedback to the user's performance.

Lieberman and Breazeal [4] use motion capture technology and provide tactile feedback for an arm movement task. Technically, their system is close to ours, as both use motion capture technology and provide vibrotactile feedback. One important difference is that our system is placed in the domain of dancing, where the whole body is moved in space to a given rhythm.

With Saltate! [3], we developed a system that supports beginners to perform a Slow Waltz in sync to the music. Saltate! detects a couples' step timings with pressure sensors and provides acoustical feedback by emphasizing the music's beats if required. Our current 
work extends our previous work and targets at another problem, learning a choreography. Yet, many design decisions were based on our experiences with Saltate!.

\section{Scope of the System}

The two most important lessons we learned from Saltate!'s evaluation are:

1) Automatically generated feedback while learning to dance can have a very high acceptance among beginners. (On a five-point Likert scale, our participants on average gave 4.31 points to the statement: This kind of support will help beginning dancers)

2) The supported task in Saltate!, dancing to the beat of the music, was too easy to learn. Additional effort should be invested into supporting more complex tasks.

The first point lead to our decision to carry on, the second showed us that we have to go from answering the question whether a step has been performed at the correct time to answering the question if it was the correct step at all. We want to be able to automatically discriminate between different steps to provide not only support but also feedback.

The most important characteristics of most dances are steps into different directions. As a starting point, we decided that we want to be able to distinguish:
1) Forward steps
2) Backward steps
3) Sideway steps
4) Closing steps

After a closing step both feet are next to each other. What we do not distinguish yet is whether a step was performed as a ball step (only the ball of a foot touches the floor) or as a full step (at one point, the whole foot is placed on the floor). We do not take into account body rotation, foot rotation, or arm movements either. These could be added in future versions of our program. Using only the mentioned steps we are still able to create choreographies of very different difficulties. In the following paragraphs, we describe different aspects of our work in detail.

\section{Technical Setup}

In order to detect our dancers' steps, we use a Vicon motion capture system. ${ }^{1}$ Motion capture data forwarded to and analyzed by our Objective-C program includes a dancer's knees and feet position. The algorithm used to detect and distinguish steps is based on each foot's velocity. If a foot stops moving we assume that a step has occurred. Based on the relative movement of the foot (compared to its last standing position and to the other foot) we are able to distinguish between forward, backward, sideway, and closing steps. The parameters and algorithms used to distinguish between different steps and their timings are still under development, we assume that the accuracy under real conditions right now is at about $95 \%$. (Wrong detections occur only sporadically while we are actively searching for weaknesses in the system's detection capabilities)

While playing music, the program concurrently analyzes steps, compares them to a stored choreography, and (if activated) provides feedback or support.

\footnotetext{
${ }^{1}$ http://www.vicon.com/products/viconmx.html
} 


\section{Experimental Setup and Evaluation}

Our experiment has the following structure:

1) Teaching session

2) Training session

3) Evaluation

4) Retention test (Two days later)

In the training session, the dancer looks at a video of a teacher performing and explaining the choreography. This is very similar to the traditional approach with a real teacher, but guarantees the same condition among all participants. In the training session, the dancer is provided with one kind of support or feedback. During the evaluation we let the dancer perform the choreography without support and feedback to several songs with rising speeds, and measure the amount of correct and incorrect steps:

A step is considered as correct if it was measured within a small time interval around the beat to which it should have been performed. We will determine the length of this interval by checking the step time differences to the music's beats of several correctly danced performances from experienced dancers. From our experience with Saltate! [3], we expect that the size of this interval will be about 250 to $300 \mathrm{~ms}$. A wrong step occurs if no correct step was detected within a time interval where a step was expected or if a step was detected outside of an expected time interval.

To measure longer term learning effects, we will execute a retention test two days after the first experimental session. In this test we will only allow a very short training session and then repeat the evaluation.

In order to minimize the effect of differently gifted persons on our results we perform the teaching, training, and evaluation session three times with each dancer - each time using a different choreography and a different support or feedback modality. We will counterbalance the order of feedback modalities and the order of choreographies.

\section{Feedback and Supporting Functions}

We want to compare a couple of different feedback and support modalities:

1) A video with a dancer performing the choreography.

2) A written representation of the choreography.

3) Vibrotactile feedback.

4) Acoustic feedback.

The video and the written representation are supporting functions: A dancer can use them if he wants to, but they are not activated automatically. They are easy to realize and are already used in dancing courses as additional help outside of course times. A benefit of video is that it is very easy to understand. The written representation requires more effort to use, but it could lead to a deeper mental processing and better memorization. 
Vibrotactile feedback is realized with small vibrational devices placed on the front, back, left, and right side of each ankle indicating the direction to which the dancer has to move. In an informal test we found that it is easy to discriminate the different vibrational devices when they are activated. We assume that this kind of feedback is easy to understand - you move into the direction where you feel the vibration. It might, however, suffer from a shift of attention away from the music towards a dancer's own body. [5]

For acoustic feedback, we plan to explore different possibilities and to include the most promising ones into our system. Current ideas include:

1) The use of stereo to indicate movements with the left or the right foot.

2) Different pitches for different steps like moving forward, backward, left, or right.

3) Different sounds for different steps.

4) Recorded speech

The first three ideas require another learning phase to be useful. This could be accomplished by playing the according sound files when the student performs the corresponding steps. Eventually, this can be combined with the initial teaching of the choreography. The third idea is based on our experience with Saltate!. There we used additional bass drums to emphasize the music's beats. Thus the overall sound experience was very natural. Our idea is to choose sounds for correct steps that fit well into the music and sounds for incorrect steps that do not.
While video and written representation are supporting functions only (and not activated automatically), vibrotactile and acoustic feedback are controlled by the system, which can control their strength (stronger or weaker vibrations, louder or softer acoustic feedback) and activation.

Vibrotactile feedback and acoustic feedback have another thing in common, as they are given while a dancer performs a dance - their exact timing is variable. We could give a hint to step back at a specific beat (to which the step should be performed) of the music or at a constant offset before that beat.

Nakamura [1] used only one offset of $500 \mathrm{~ms}$ ahead of a desired movement, but it is obvious that the timing has an effect: If a hint for a specific movement is given concurrently to that movement, a student cannot prepare for this movement. If it is too far ahead, it might feel unnatural or he/she is still busy executing a prior movement. Saltate!'s feedback was played at the beats of the music. However, its purpose was to support the dancing in sync to the music, not to support the learning of a choreography based on several different steps.

\section{Choreography Development}

The choreography used in our experiment has to fulfill two main requirements:

1) Its features must be detectable by our system, i.e., it must consists of the steps described before but no additional steps or rotations. 


\begin{tabular}{|r|c|c|}
\hline Beat & Left Foot & Right Foot \\
\hline 1 & left & \\
\hline 2 & close & \\
\hline 3 & & go ahead \\
\hline 4 & & close \\
\hline 5 & & right \\
\hline 6 & & close \\
\hline 7 & left & \\
\hline 8 & & close \\
\hline 9 & step back & \\
\hline 10 & & close \\
\hline 11 & left & \\
\hline 12 & & close \\
\hline
\end{tabular}

Figure 1. A written representation of one of our short choreographies.
2) Its difficulty must be well chosen. If it is too high we would require too much time to evaluate effects, if it is too easy and all of our dancers perform without mistakes, there are no differences we could analyze.

We iteratively created three different short choreographies of twelve beats length. We trained the initial choreographies ourselves and taught them to volunteers, refining the choreographies based on the difficulty our volunteers experienced to learn them. The speed of the music we used ranged from 30 to 42 bars per minute (measured in $4 / 4$ time)

\section{Acknowledgements}

This work was funded in part by the German B-IT Foundation.

\section{References}

[1] Akio Nakamura, Sou Tabata, Tomoya Ueda, Shinichiro Kiy- ofuji, and Yoshinori Kuno. Multimodal presentation method for a dance training system. In $\mathrm{CHI}$ '05: $\mathrm{CHI}$ '05 extended abstracts on Human factors in computing systems, pages 1685-1688, New York, NY, USA, 2005. ACM.

[2] Daniel Spelmezan, Adalbert Schanowski, Jan Borchers. Wearable Automatic Feedback Devices for

\section{Summary}

We described a system that supports learning of simple dance choreographies and an experiment to evaluate and compare four types of learning support for dancing: The acoustic and vibrotactile feedback of our system, video, and a textual representation.

Physical Activities. In Proceedings of the $4^{\text {th }}$ International Conference on Body Area Networks, LNICST, Los Angeles, CA, USA, April 2009. ICST.

[3] Dieter Drobny, Malte Weiss, Jan Borchers. Saltate! a sensor-based system to support dance beginners, $\mathrm{CHI}$ '09 extended abstracts on Human factors in computing systems, pages 3943-3948, April 04-09, 2009, Boston, MA, USA. ACM.

[4] Jeff Lieberman, Cynthia Breazeal. TIKL:

Development of a Wearable Vibrotactile Feedback Suit for Improved Human Motot Learning, IEEE Transactions on Robotics, Vol. 23, No. 5, October 2007.

[5] Gabriele Wulf. Attention and Motor Skill Learning. Human Kinetics, 2007. 